\title{
Time-Dependent Scattering Functions of Rouse-Zimm Polymer Chains
}

\author{
José A. EsCUdERo and Juan J. FreIRE* \\ Departamento de Quimica Física, Facultad de Ciencias Quimicas, \\ Universidad Complutense, Madrid-3, Spain.
}

(Received October 19, 1981)

\begin{abstract}
Time-dependent scattering functions have been numerically calculated from a rigorous version of the Rouse-Zimm theory for different values of the scattering variable, the chain length and the hydrodynamic interaction. These functions can be directly related to measurements of quasielastic light scattering from flexible polymers in solution. The results are analysed and fitted to simple functions commonly employed in the treatment of experimental data. Changes in the values of the fitting parameters with chosen time ranges are studied. A description of the scattering functions from the theoretical values of their first, second, and third derivatives at short times is considered and discussed. Also, a simple approximate formula is derived. This formula gives a reasonably good description of the exact results for many realistic cases.
\end{abstract}

KEY WORDS Scattering Functions /Polymer Chains /Rouse-Zimm /

Polarized quasielastic light scattering measurements from flexible polymers in solution are now frequently made since they provide useful information on the translational motion and the internal dynamics of polymer chains. ${ }^{1-5}$ For small values of the scattering variable, $q=|\boldsymbol{q}|$, this technique yields a direct estimation of the translational diffusion coefficient. However, the internal motions intervene for higher values of $q$ and presentation of the information takes on a somewhat more complicated form. ${ }^{1}$ In order to compare the experimental timecorrelation function of the polarized scattered light intensity from a chain composed of $N+1$ identical isotropic scattering centers with theoretical calculations, the "intermediate scattering function" is defined as

$$
P(\boldsymbol{q}, t)=\left\langle\sum_{i=0}^{N} \sum_{j=0}^{N} \exp \left\{i \boldsymbol{q} \cdot\left[\boldsymbol{r}_{i}{ }^{0}-\boldsymbol{r}_{j}{ }^{0}(t)\right]\right\}\right\rangle
$$

where $\boldsymbol{r}_{i}^{0}$ and $\boldsymbol{r}_{j}^{0}$ are the positions of the scattering centers $i$ and $j$ at times zero and $t$ with respect to an external frame.

In a typical homodyne experiment with a digital autocorrelator, the normalized intensity correlation, $g^{(2)}(t)$, is related with $P(\boldsymbol{q}, t)$ through the

\footnotetext{
* To whom correspondence should be addressed.
}

relations

$$
\begin{gathered}
g^{(2)}(t)=1+\left[g^{(1)}(t)\right]^{2} \\
g^{(1)}(t) \propto P(q, t) .
\end{gathered}
$$

The traditional treatment for the functions $P(q, t)$ obtained from the experiments has been to fit them to the sum of a few exponentials. These fittings provide a set of parameters that can be interpreted in terms of molecular motions.

From a theoretical point of view, the most usual representation of the dynamics of a flexible polymer is provided by the Rouse-Zimm model ${ }^{6}$ applicable to the low frequency behaviour of Gaussian chains. This model considers hydrodynamic interactions between statistical units and predicts a multiexponential form for scattering functions. Due to the complexity of the calculations only a few numerical results for $P(q, t)$ have been obtained..$^{1,7-10}$ Some of these results are restricted to small values of $N^{8}$ or small values of $x^{7}$, the reduced scattering parameter, defined by,

$$
x=q^{2}\left\langle s^{2}\right\rangle,
$$

where $\left\langle s^{2}\right\rangle$ is the mean quadratic radius of gyration of the chain. Other calculations, ${ }^{1,9,10}$ 


\section{J. A. Escudero and J. J. Freire}

though more extensive, are biased by several approximations.

Here, we perform a series of exact calculations for the intermediate scattering functions of RouseZimm chains, over a wide range of values for $x$ and $N$. Furthermore, we study several types of fitting for these functions over different time intervals to ascertain the reproducibility of fitting parameters.

Our calculations are also useful in testing an alternative approach based on the study of the short-time first derivatives of $P(\boldsymbol{q}, t)$ that can be obtained from the initial slopes in representations of $\ln P(\boldsymbol{q}, t) v s$. time. ${ }^{11}$ As previously shown,${ }^{11,12}$ these slopes describe the whole function for $x \rightarrow 0$ or $x / N>20$. However, in the intermediate cases, corresponding to the highest accessible values of $x$ in light scattering experiments with long flexible chains, the initial slopes can only describe a limited region of the experimental time interval. With our numerical results we study the length of this region and discuss the applicability of the method to different cases.

We also discuss the possibility of inferring the actual shape of the scattering function from four parameters related to the theoretical values of its first three derivatives for short times. Our approach is somewhat parallel, though formally simpler, to the study of memory effects in the projection operator formalism for the scattering function, proposed recently by Dahler and coworkers, ${ }^{13,14}$ yielding similar results.

Finally, we derive an approximate formula which gives accurate results for the scattering function when sufficiently large values of the combined variable $x / t$ are considered.

\section{THEORETICAL EXPRESSIONS}

The aim of this Section is to review or establish the different expressions needed for the numerical calculation described in Sections II and III.

Pecora ${ }^{15}$ obtained an exact expression for the scattering function from eq 1 by solving the diffusion (Fokker-Planck) equation of the chain. It can be written as

$$
\begin{aligned}
P(\boldsymbol{q}, t)= & (N+1)^{-2} \sum_{i=0}^{N} \sum_{j=0}^{N} \exp \left\{-\frac{1}{6} q^{2} \sum_{k=0}^{N} b^{2} \mu_{k}^{-1}\left(Q_{i k}^{2}\right.\right. \\
& \left.\left.+Q_{j k}^{2}-2 Q_{i k} Q_{j k} \mathrm{e}^{-t / \tau}\right)\right\}
\end{aligned}
$$

where $b$ is the length of the model statistical unit. Other quantities in eq 5 are defined by Zimm's matrix equation ${ }^{6}$

$$
\boldsymbol{H} \boldsymbol{A} \boldsymbol{\alpha}_{k}=\lambda_{k} \boldsymbol{\alpha}_{k} .
$$

Thus, $Q$ is a transform matrix whose columns are the eigenvectors $\boldsymbol{\alpha}_{k}$. The eigenvalues are the non-null elements of the diagonal matrix $\boldsymbol{A}$,

$$
\boldsymbol{\Lambda}=\boldsymbol{Q}^{-1} \boldsymbol{H} \boldsymbol{A} \boldsymbol{Q},
$$

while $\mu_{k}$ represents the non-null elements of the diagonal matrix ${ }^{6} \boldsymbol{M}$,

$$
M=Q^{T} A Q .
$$

$\tau_{k}$ is the relaxation time related to the eigenvalue $\lambda_{k}$ by

$$
\tau_{k}=b^{2} f / 3 K_{\mathrm{B}} T \lambda_{k},
$$

where $f$ is the friction constant of the statistical unit and $K_{\mathrm{B}} T$ is the Boltzmann factor. Matrices $\boldsymbol{H}$ and $\boldsymbol{A}$ in eq 6 are defined in the Rouse-Zimm theory. ${ }^{6}$ The elements of $\boldsymbol{A}$ are

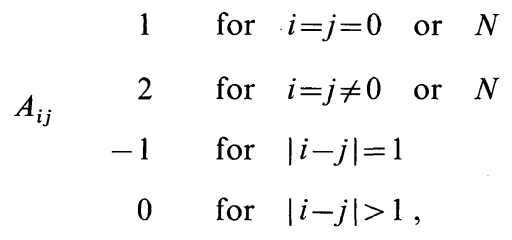

while the elements of $\boldsymbol{H}, H_{i j}$, depend on the hydrodynamic interaction parameter $h^{*}$,

$$
\begin{gathered}
H_{i j}=1 \quad \text { for } \quad i=j \\
H_{i j}=(\pi / 3)^{1 / 2}\left\langle R_{i j}^{-1}\right\rangle h^{*} b \quad i \neq j
\end{gathered}
$$

where $\left\langle R_{i j}^{-1}\right\rangle$ is the mean reciprocal distance between units $i$ and $j$. For a Gaussian chain, eq $11 \mathrm{~b}$ can be written as

$$
H_{i j}=2^{1 / 2} h^{*}|i-j|^{-1 / 2} .
$$

Therefore, the scattering function depends on the variables $t$ and $q$, and several other parameters. For our convenience, we reduce the function $P$ so that it depends only on two experimental variables and two theoretical parameters. With this purpose in mind, we introduce

$$
T_{\mathrm{R}}=1 / D_{\mathrm{z}} q^{2},
$$

where $D_{z}$ is the theoretical translational diffusion coefficient of the Rouse-Zimm theory, ${ }^{16}$ 


$$
D_{z}=(N+1)^{-1}\left(K_{\mathrm{B}} T / f\right) v_{0},
$$

$v_{0}$ is the $(0,0)$ term of the diagonal matrix ${ }^{6} N$,

$$
N=Q^{-1} H Q^{-1 T}
$$

Noticing that $Q_{i 0}=(N+1)^{-1 / 2}$ for any $i$, we get

$$
D_{\mathrm{z}}=\left(K_{\mathrm{B}} T / f\right)\left[\sum_{i=0}^{N} \sum_{j=0}^{N}\left(\boldsymbol{H}^{-1}\right)_{i j}\right]^{-1},
$$

which is consistent with the result obtained from the Kirkwood theory (version 1948) ${ }^{17}$ with a preaveraged Ossen tensor. Therefore, if $H_{i j}$ is calculated from eq $11 \mathrm{a}$ and $11 \mathrm{~b}$, eq 15 is valid for any chain statistics.

We also use the known formula for the mean quadratic radius of gyration of a Gaussian chain,

$$
\left\langle s^{2}\right\rangle=\frac{1}{6} N_{\mathrm{G}} b^{2}
$$

where

$$
N_{\mathrm{G}}=N\left[1+(N+1)^{-1}\right] \text {. }
$$

Then, with eq $4,9,12$, and 16 and a previous evaluation of the $k=0$ term $^{11}$ of eq 5 , the scattering function can be expressed as

$$
\begin{aligned}
P\left(x, t / T_{\mathrm{R}}\right) & =(N+1)^{-2} \mathrm{e}^{-\left(t / T_{\mathrm{R}}\right)} \sum_{i=0}^{N} \sum_{j=0}^{N} \\
& \times \exp \left[-\left(x / N_{\mathrm{G}}\right) \sum_{k=1}^{N} \mu_{k}^{-1}\left\{Q_{i k}^{2}+Q_{j k}^{2}\right.\right. \\
& \left.\left.-2 Q_{i k} Q_{j k} \exp \left[-\lambda_{k}\left(t / T_{\mathrm{R}}\right) N_{\mathrm{G}} / 2 x D^{*}\right]\right\}\right]
\end{aligned}
$$

with

$$
D^{*}=D_{\mathrm{z}} f / K_{\mathrm{B}} T .
$$

Equation 18 allows us to obtain numerical results for $P$ for any set of values of $N, x, t / T_{\mathrm{R}}$ and $h^{*}$.

On the other hand, from eq 5 and the well known result that

$$
\sum_{k=0}^{N} b^{2} \mu_{k}^{-1}\left(Q_{i k}-Q_{j k}\right)^{2}=b^{2}|i-j|,
$$

we can obtain ${ }^{11}$ the short-time first derivative of $P$,

$$
\lim _{t \rightarrow 0} \frac{\mathrm{d} P(q, t)}{\mathrm{d} t}
$$

$$
=-(N+1)^{-2}\left(q^{2} K_{\mathrm{B}} T / f\right) \sum_{i=0}^{N} \sum_{j=0}^{N} \mathrm{e}^{-(1 / 6) q^{2} b^{2}|i-j|} H_{i j},
$$

or, in terms of our practical variables,

$$
\begin{aligned}
D_{1} & \equiv \lim _{t / T_{\mathrm{R}} \rightarrow 0} \frac{\mathrm{d} P\left(x, t / T_{\mathrm{R}}\right)}{\mathrm{d}\left(t / T_{\mathrm{R}}\right)} \\
& =-(N+1)^{-2}\left(1 / D^{*}\right) \sum_{i=0}^{N} \sum_{j=0}^{N} \mathrm{e}^{-x|i-j| / N_{\mathrm{G}}} H_{i j},
\end{aligned}
$$

Also, we introduce here the magnitude $D_{\text {app }} / D_{z}$ defined by

$$
D_{\text {app }} / D_{\mathrm{z}}=-P^{-1}(x) D_{1},
$$

where $P(x)$ is the value of the scattering function at time zero, or the form factor, that can be evaluated from

$$
P(x) \equiv P(q, 0)=(N+1)^{-2} \sum_{i=0}^{N} \sum_{j=0}^{N} \mathrm{e}^{-|i-j| x / N_{\mathrm{G}}} .
$$

When experiments are performed with the homodyne detection technique, eq 2 shows that we should deal with squared scattering functions. Particularly, representations ${ }^{2}$ of $\ln \left[P\left(x, t / T_{\mathrm{R}}\right) /\right.$ $P(x)]^{2}$ vs. $t / T_{\mathrm{R}}$ have an initial slope, $P_{\mathrm{D}}$, directly related $^{10}$ to $D_{\text {app }} / D_{\mathrm{z}}$,

$$
P_{\mathrm{D}} \equiv \lim _{t / T_{\mathrm{R}} \rightarrow 0} \frac{\mathrm{d} \ln \left[P\left(x, t / T_{\mathrm{R}}\right) / P(x)\right]^{2}}{\mathrm{~d}\left(t / T_{\mathrm{R}}\right)}=-2 D_{\text {app }} / D_{\mathrm{z}} .
$$

Short-time higher derivatives of $P\left(x, t / T_{\mathrm{R}}\right)$ should be difficult to measure. However, as explained in the Introduction, we are interested in theoretical values of these derivatives in order to analyze the range of times for which the derivatives describe the form of the function. Equations 5 and 20 lead to the following results,

$$
\begin{aligned}
\lim _{t \rightarrow 0} \frac{\mathrm{d}^{2} P(q, t)}{\mathrm{d} t^{2}} \\
=(N+1)^{-2}\left(q^{2} K_{\mathrm{B}} T / f\right)^{2} \sum_{i=0}^{N} \sum_{j=0}^{N} \mathrm{e}^{-(1 / 6) q^{2} b^{2}|i-j|} \\
\times\left[\left(\sum_{k=0}^{N} \mu_{K}{ }^{-1} Q_{i K} Q_{j K} \lambda_{K}\right)^{2}\right. \\
\left.+3(q b)^{-2} \sum_{k=0}^{N} \mu_{K}{ }^{-1} Q_{i K} Q_{j K} \lambda_{K}{ }^{2}\right]
\end{aligned}
$$




\section{J. A. Escudero and J. J. Freire}

and

$$
\begin{aligned}
\lim _{t \rightarrow 0} \frac{\mathrm{d}^{3} P(q, t)}{\mathrm{d} t^{3}} & -(N+1)^{2}\left(q^{2} K_{\mathrm{B}} T / f\right)^{3} \sum_{i=0}^{N} \sum_{j=0}^{N} \mathrm{e}^{-(1 / 6) q^{2} b^{2}|i-j|} \\
= & \times\left[\left(\sum_{k=0}^{N} \mu_{K}{ }^{-1} Q_{i K} Q_{j K} \lambda_{K}\right)^{3}+9(q b)^{-2}\right. \\
& \times\left(\sum_{k=0}^{N} \mu_{K}{ }^{-1} Q_{i K} Q_{j K} \lambda_{K}\right)\left(\sum_{k=0}^{N} \mu_{K}{ }^{-1} Q_{i K} Q_{j K} \lambda_{K}{ }^{2}\right) \\
& \left.+9(q b)^{-4} \sum_{k=0}^{N} \mu_{K}{ }^{-1} Q_{i K} Q_{j K} \lambda_{K}{ }^{3}\right] .
\end{aligned}
$$

In previous work ${ }^{11}$ we derived the relation

$$
\sum_{k=0}^{N} \mu_{K}{ }^{-1} Q_{i K} Q_{j K} \lambda_{K}=H_{i j}
$$

which can be easily generalized since for any integer $n$,

$$
\sum_{k=0}^{N} \mu_{K}{ }^{-1} Q_{i K} Q_{j K} \lambda_{K}{ }^{n}=\left[\left(\boldsymbol{Q} \Lambda Q^{-1}\right)^{n-1} \boldsymbol{Q} N Q^{T}\right]_{i j} .
$$

Then, eq 7, 14, and 29 give

$$
\sum_{k=0}^{N} \mu_{K}{ }^{-1} Q_{i K} Q_{j K} \lambda^{n}=\left[(\boldsymbol{H} \boldsymbol{A})^{n-1} \boldsymbol{H}\right]_{i j}
$$

Considering also eq $4,12,16$, and 19 , we obtain the following expressions for the derivatives as functions of the practical variables,

$$
\begin{aligned}
& D_{2} \equiv \lim _{t / T_{\mathrm{R}} \rightarrow 0} \frac{\mathrm{d}^{2} P\left(x, t / T_{\mathrm{R}}\right)}{\mathrm{d}\left(t / T_{\mathrm{R}}\right)^{2}} \\
&=(N+1)^{-2}\left(1 / D^{*}\right)^{2} \sum_{i=0}^{N} \sum_{j=0}^{N} \mathrm{e}^{-x|i-j| / N_{\mathrm{G}}} \\
& \times\left[H_{i j}^{2}+\left(N_{\mathrm{G}} / 2 x\right)(\boldsymbol{H} \boldsymbol{A} \boldsymbol{H})_{i j}\right]
\end{aligned}
$$

and

$$
\begin{aligned}
D_{3} & \equiv \lim _{t / T_{\mathrm{R}} \rightarrow 0} \frac{\mathrm{d}^{3} P\left(x, t / T_{\mathrm{R}}\right)}{\mathrm{d}\left(t / T_{\mathrm{R}}\right)^{3}} \\
& =-(N+1)^{-2}\left(1 / D^{*}\right)^{3} \sum_{i=0}^{N} \sum_{j=0}^{N} \mathrm{e}^{-x|i-j| / N_{G}}
\end{aligned}
$$

$$
\begin{aligned}
& \times\left[H_{i j}^{3}+\left(3 N_{\mathrm{G}} / 2 x\right) H_{i j}(\boldsymbol{H} \boldsymbol{A} \boldsymbol{H})_{i j}\right. \\
& \left.+\left(N_{\mathrm{G}} / 2 x\right)^{2}(\boldsymbol{H} \boldsymbol{A} \boldsymbol{H} \boldsymbol{A} \boldsymbol{H})_{i j}\right] .
\end{aligned}
$$

A similar procedure would easily provide corresponding expressions for higher derivatives.

\section{THE SCATTERING FUNCTION}

In this section we report the numerical results obtained for the scattering function and the values of the parameters obtained from their fittings to simple functions of different forms.

In order to evaluate the scattering function from eq 18 , the eigenvalues and eigenvectors of eq 6 must be calculated. Methods for solving eq 6 have been extensively reported by several authors ${ }^{8,18,19}$ in recent years (see ref 20 for a detailed discussion of those methods). We have found, however, that results can be obtained more directly by using a standard FORTRAN subroutine, developed some time ago. This method allows determination of eigenvalues and eigenvectors of the product $\boldsymbol{M}_{1}^{-1} \cdot \boldsymbol{M}_{2}$, where $\boldsymbol{M}_{1}$ and $\boldsymbol{M}_{2}$ are two symmetric matrices, following a procedure similar to that proposed by Lin and Schurr, ${ }^{8}$ but including the calculation of $\boldsymbol{H}^{-1}$. This is not inconvenient, since we also use $\boldsymbol{H}^{-1}$ to obtain $D_{z}$ and $D^{*}$ from eq 15 in a rigorous evaluation of the scattering function with our practical variables.

The accuracy of our numerical resolution of eq 6 was tested in several ways. First, we compared the eigenvalues and eigenvectors obtained in the free draining limit (i.e. with $h^{*}=0$ ) with those calculated from the following analytical formulas developed in this particular case, ${ }^{5,8}$

$$
\begin{gathered}
\lambda_{k}=4 \sin ^{2}[\pi k / 2(N+1)], \quad k=0, \cdots, N \\
Q_{i j}=N_{\mathrm{Q}} \cos [\pi(i+1 / 2) j /(N+1)], \\
i, j=0, \cdots, N
\end{gathered}
$$

with

$$
\begin{array}{cc}
N_{\mathrm{Q}}=(N+1)^{-1 / 2} & \text { if } j=0 \\
N_{\mathrm{Q}}=[2 /(N+1)]^{1 / 2} & \text { if } j \neq 0 .
\end{array}
$$

The numerical results obtained with the diagonalization subroutine are consistent with the results obtained from these equations. Moreover, we also reproduced the eigenvalues and eigenvectors calcu- 
lated by Perico et al. ${ }^{18}$ for $N=100$ and several values of $h^{*}$.

From the eigenvalues and eigenvectors and eq 15, 17,18 , and 19 , we obtained the scattering functions for different sets of the variables.

Figures 1 and 2 show the results for $r_{\mathrm{p}}=$ $\left[\ln P\left(t / T_{\mathrm{R}}\right) / P(x)\right]^{2}$ vs. $t / T_{\mathrm{R}}$ corresponding to $N=10$ and $50, h^{*}=0.25$ (the most adequate value ${ }^{11}$ for a non-draining Gaussian chain) and several values of $x$. For small $x$ these functions show a weak dependence on $N$. However, when $x \gg N$, the dependence is strong, and $r_{\mathrm{p}}$ undergoes a noticeable decrease as $N$ increases. In fact, we obtained a large set of results with different values of $N$ and $x$. The corresponding tables are too lengthly to be reproduced here, but are available upon request. ${ }^{22} \mathrm{We}$ compared these results with those obtained previously by one of $\mathrm{us}^{10}$ for $N>20$, using approximate expressions for the eigenvalues and eigenvectors valid for high values of $N$. Though differences between both kinds of results are smaller than $3 \%$ in all cases, we think that it is worth performing an exact diagonalization since in many cases its performance is faster than that of the triple sum over $N$ involved in eq 18.

The broken straight lines in Figures 1 and 2 have been plotted with a slope equal to the theoretical values for $p_{\mathrm{D}}$ calculated from eq 22 to 25 . According to eq 25 these straight lines should represent the initial single exponential behaviour of the scattering functions, as apparent from the figures. It can be observed that this behaviour extends to the whole time range over the limits $x \approx 0, x / N \gg 1$ (or $q^{2} b^{2} \gg 1$ ) as was pointed out earlier. ${ }^{8,11}$

For the intermediate values of $x$, however, the initial behavior continues only within a very small time range. Nevertheless, one can extract the initial slopes from the theoretical curves in Figures 1 and 2 with reasonable accuracy. This confirms the procedure utilized in previous work for obtaining slopes from the experimental data. ${ }^{10,11}$

In order to ascertain the correctness of the usual treatment of the experimental scattering functions, we carried out several least-squares fittings for our

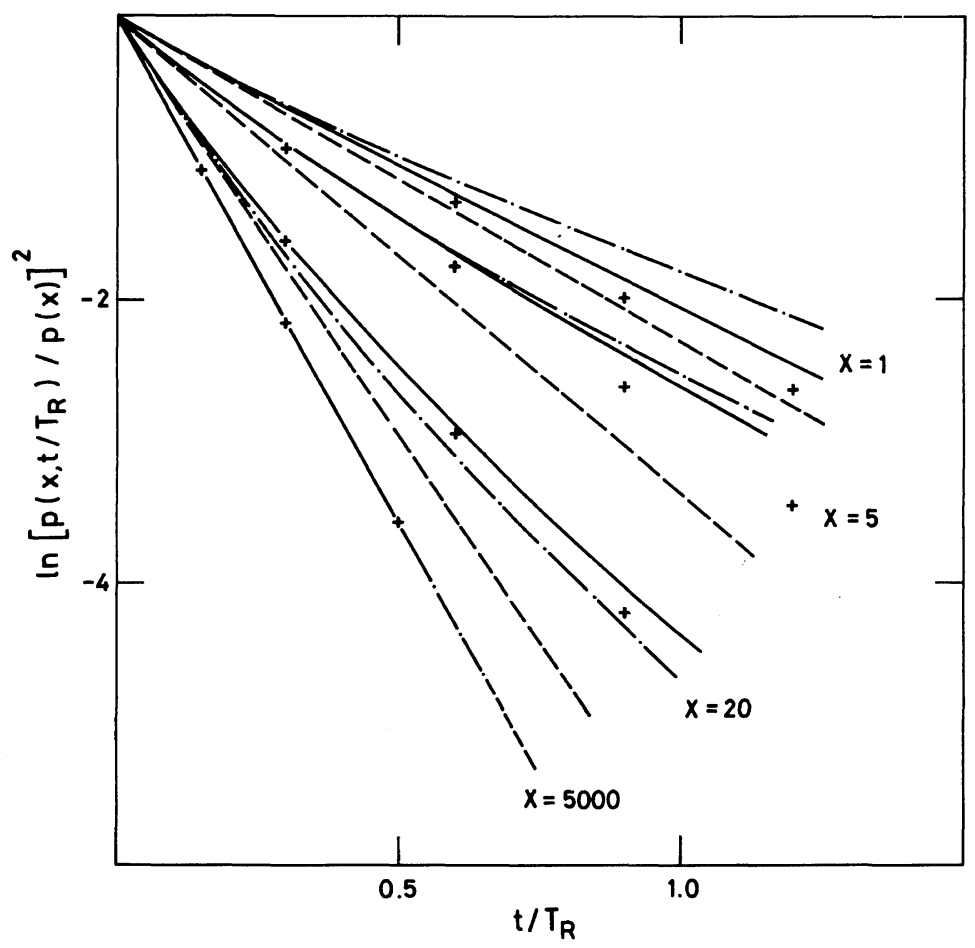

Figure 1. Logarithmic plots of normalized scattering functions versus the reduced time variable for $N=$ 10 and $h^{*}=0.25$. (-) exact results; (---) initial slope single exponentials; (- $-{ }_{-} \cdot-$ ) results from eq $47 ;(+)$ results obtained from the first three theoretical initial derivatives. 


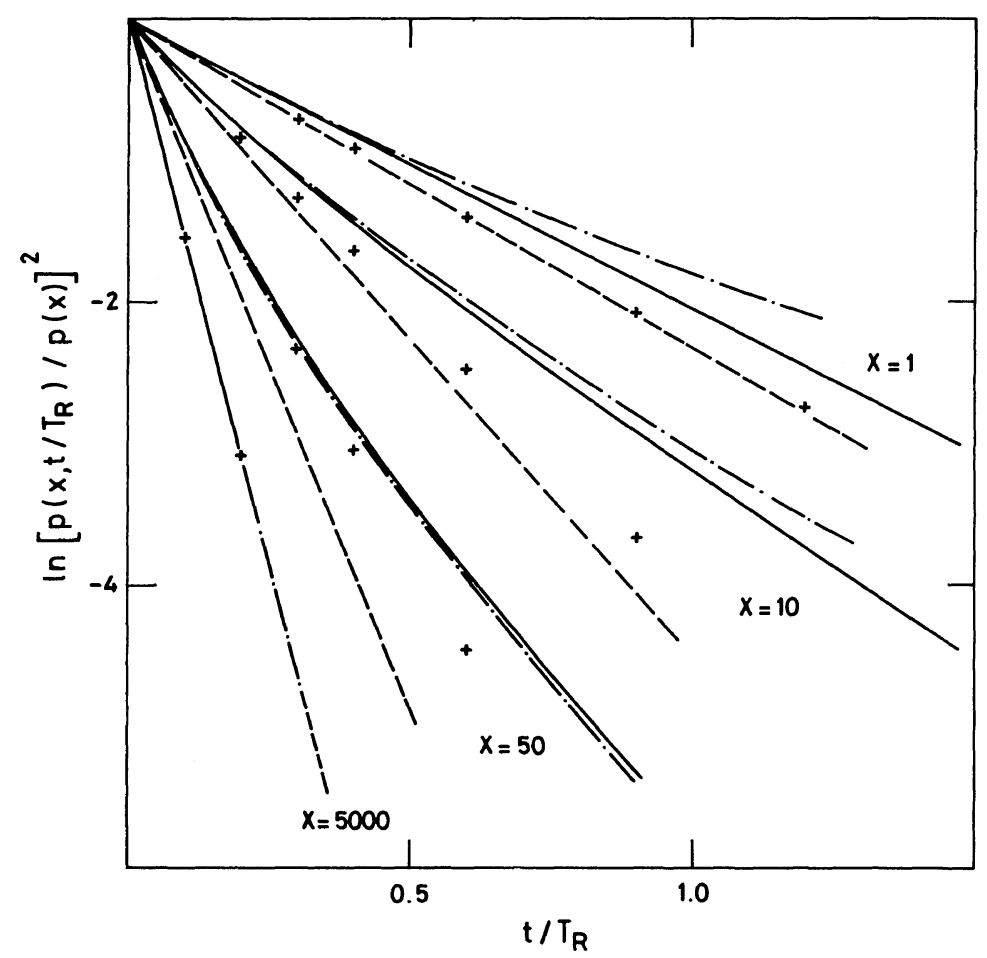

Figure 2. As Figure 1 but with $N=50$.

theoretical functions, considering different intervals of $t / T_{\mathrm{R}}$ defined from $t / T_{\mathrm{R}}=0$ to $t / T_{\mathrm{R}}=\Delta\left(t / T_{\mathrm{R}}\right)$. The deviations of these fittings are evaluated as

$$
d=\left\{\sum_{i=1}^{m}\left\{y_{i}-\left[y\left(t / T_{\mathrm{R}}\right)\right]_{i}\right\}^{2} / m\left(m-m_{\mathrm{p}}\right)\right\}^{1 / 2}
$$

where $y_{i}$ is the value of the function to be fitted at point $\left(t / T_{\mathrm{R}}\right)_{i},\left[y\left(t / T_{\mathrm{R}}\right)\right]_{i}$ is the value obtained with the fitting function at the same point, $m$ is the number of points involved in the fitting, which uses $m_{\mathrm{p}}$ parameters. We have considered the following points: $\left(t / T_{\mathrm{R}}\right)=10^{-5}, 0.01,0.05,0.1,0.3,0.6,0.9,1.2$, and 1.5 , taking for each value of $\Delta\left(t / T_{\mathrm{R}}\right)$ those of the points included within the corresponding interval. First we fitted the scattering functions to single exponentials,

$$
y=P\left(x, t / T_{\mathrm{R}}\right) / P(x)=\mathrm{e}^{-\mathrm{K}\left(t / T_{\mathrm{R}}\right)} .
$$

The parameter $K$ corresponds to $D_{\text {app }} / D_{\mathrm{z}}$ for sufficiently small values of $t / T_{\mathrm{R}}$. The results are given in Table I, and Table II shows the corresponding deviations.

It should be considered that, though deviations are small and, in general, could be acceptable from an experimental point of view, the chosen range of time influences strongly the value of $K$ for intermediate $x$. Thus, in order to assert the reliability of any parameter fitted from experimental data of the scattering function, one must be sure of the constancy of its value throughout different realistic intervals of time, even when small deviations are found.

A fitting function of the type,

$$
y=\ln \left[P\left(x, t / T_{\mathrm{R}}\right) / P(x)\right]=-K^{\prime}\left(t / T_{\mathrm{R}}\right)
$$

has been also investigated. We obtained numerical values of $K^{\prime}$ which, in general, are close to those of $K$. In any case, the results are much more sensitive to the selected time interval than to the choice of the fitting method for these two types. We also considered a double exponential fitting of the form

$$
\begin{aligned}
y= & P\left(x, t / T_{\mathrm{R}}\right) / P(x)=P_{1} \mathrm{e}^{-K_{1}\left(t / T_{\mathrm{R}}\right)} \\
& +P_{2} \mathrm{e}^{-K_{2}\left(t / T_{\mathrm{R}}\right)},
\end{aligned}
$$

whose results are presented in Table III. It is shown 
Table I. Results for $K$ (parameter of the single exponential fitting). The values for $\Delta\left(t / T_{\mathrm{R}}\right)=0$ were obtained from the short-time theoretical first derivatives

\begin{tabular}{rrrrrr}
\hline & & \multicolumn{5}{c}{$\Delta\left(t / T_{\mathrm{R}}\right)$} \\
\cline { 3 - 6 }$N$ & $x$ & 0 & 0.1 & 0.9 & 1.5 \\
\hline 2 & 1 & 1.154 & 1.125 & 1.058 & 1.045 \\
2 & 10 & 1.799 & 1.787 & 1.730 & 1.709 \\
2 & 20 & 1.835 & 1.829 & 1.804 & 1.795 \\
2 & 100 & 1.836 & 1.836 & 1.835 & 1.835 \\
& & & & & \\
10 & 1 & 1.151 & 1.105 & 1.043 & 1.032 \\
10 & 10 & 2.268 & 2.142 & 1.846 & 1.786 \\
10 & 20 & 2.979 & 2.862 & 2.560 & 2.518 \\
10 & 1000 & 3.580 & 3.577 & 3.572 & 3.572 \\
& & & & & \\
50 & 1 & 1.153 & 1.104 & 1.041 & 1.031 \\
50 & 10 & 2.254 & 2.060 & 1.779 & 1.722 \\
50 & 20 & 3.110 & 2.794 & 2.402 & 2.354 \\
50 & 1000 & 7.727 & 7.675 & 7.630 & 7.630 \\
\hline
\end{tabular}

Table II. Values of $\mathrm{d}$ (deviations) for single exponential fittings

\begin{tabular}{rrrcc}
\hline & & \multicolumn{3}{c}{$\Delta\left(t / T_{\mathrm{R}}\right)$} \\
\cline { 3 - 5 }$N$ & $x$ & 0.1 & 0.9 & 1.5 \\
\hline 2 & 1 & $1.9 \times 10^{-4}$ & $1.8 \times 10^{-3}$ & $1.8 \times 10^{-3}$ \\
2 & 10 & $8.6 \times 10^{-5}$ & $1.8 \times 10^{-3}$ & $2.3 \times 10^{-3}$ \\
2 & 20 & $3.9 \times 10^{-5}$ & $8.1 \times 10^{-4}$ & $1.0 \times 10^{-3}$ \\
2 & 1000 & $7.6 \times 10^{-7}$ & $1.7 \times 10^{-5}$ & $2.1 \times 10^{-5}$ \\
& & & & \\
10 & 1 & $2.5 \times 10^{-4}$ & $1.5 \times 10^{-3}$ & $1.5 \times 10^{-3}$ \\
10 & 10 & $8.0 \times 10^{-4}$ & $7.1 \times 10^{-3}$ & $7.6 \times 10^{-3}$ \\
10 & 20 & $7.6 \times 10^{-4}$ & $7.2 \times 10^{-3}$ & $7.2 \times 10^{-3}$ \\
10 & 1000 & $1.5 \times 10^{-5}$ & $1.3 \times 10^{-4}$ & $1.2 \times 10^{-4}$ \\
& & & & \\
50 & 1 & $2.5 \times 10^{-4}$ & $1.5 \times 10^{-3}$ & $1.5 \times 10^{-3}$ \\
50 & 10 & $1.0 \times 10^{-3}$ & $6.6 \times 10^{-3}$ & $7.2 \times 10^{-3}$ \\
50 & 20 & $1.3 \times 10^{-3}$ & $8.2 \times 10^{-3}$ & $8.0 \times 10^{-3}$ \\
50 & 1000 & $2.8 \times 10^{-4}$ & $6.1 \times 10^{-4}$ & $4.8 \times 10^{-4}$ \\
\hline
\end{tabular}

Table III. Results of fittings to the sum of two exponentials

\begin{tabular}{|c|c|c|c|c|c|c|c|c|c|c|}
\hline \multirow{2}{*}{$N$} & \multirow{2}{*}{$x$} & \multirow[b]{2}{*}{$\Delta\left(t / T_{\mathrm{R}}\right)$} & \multicolumn{2}{|c|}{$P_{1}$} & \multicolumn{2}{|c|}{$P_{2}$} & \multicolumn{2}{|c|}{$K_{1}$} & \multicolumn{2}{|c|}{$K_{2}$} \\
\hline & & & 0.3 & 1.5 & 0.3 & 1.5 & 0.3 & 1.5 & 0.3 & 1.5 \\
\hline 2 & 1 & & 0.974 & 0.967 & 0.026 & 0.032 & 1.012 & 1.002 & 6.481 & 5.541 \\
\hline 2 & 10 & & 0.596 & 0.512 & 0.404 & 0.488 & 1.362 & 1.271 & 2.444 & 2.300 \\
\hline 2 & 20 & & 0.589 & 0.502 & 0.411 & 0.498 & 1.536 & 1.485 & 2.262 & 2.186 \\
\hline 2 & 1000 & & 0.889 & 0.511 & 0.111 & 0.488 & 1.694 & 1.786 & 1.853 & 1.887 \\
\hline 10 & 1 & & 0.986 & 0.977 & 0.014 & 0.023 & 1.018 & 1.000 & 9.554 & 6.480 \\
\hline 10 & 10 & & 0.859 & 0.730 & 0.141 & 0.270 & 1.549 & 1.334 & 6.621 & 4.543 \\
\hline 10 & 20 & & 0.707 & 0.573 & 0.293 & 0.426 & 1.901 & 1.656 & 5.569 & 4.668 \\
\hline 10 & 1000 & & 0.522 & 0.523 & 0.478 & 0.477 & 3.356 & 3.336 & 3.824 & 3.802 \\
\hline 50 & 1 & & 0.986 & 0.979 & 0.014 & 0.021 & 1.018 & 1.000 & 10.21 & 7.035 \\
\hline 50 & 10 & & 0.918 & 0.744 & 0.082 & 0.255 & 1.610 & 1.312 & 8.804 & 4.370 \\
\hline 50 & 20 & & 0.885 & 0.705 & 0.114 & 0.294 & 2.096 & 1.708 & 10.33 & 5.686 \\
\hline 50 & 1000 & & 0.503 & 0.557 & 0.497 & 0.443 & 8.846 & 8.714 & 6.594 & 6.479 \\
\hline
\end{tabular}

that for $x \approx 0$, one of the exponentials is clearly predominant, while for $x / N \gg 1$, the two exponentials tend to be similar in weight and shape. Behaviour of this kind was predicted by Dahler and coworkers through the projection operator formalism for a free-draining limit. ${ }^{13}$ Our fittings indicate that this behaviour is also followed for $h^{*}=$ 0.25 , though those authors predict a predominant exponential for any value of $x$ in the non-draining cases. $^{14}$

Table IV shows the deviations obtained with this fitting. As expected, they are in general significantly smaller than those obtained in the single exponential fitting, though variations in the value of the parameters with the time range were found, as can be observed from the results presented in Table III. It should be pointed out that these variations with time range for all the different types of fittings 
Table IV. Deviations of the fittings to the sum of two exponentials

\begin{tabular}{|c|c|c|c|}
\hline \multirow{2}{*}{$N$} & \multirow{2}{*}{$x$} & \multicolumn{2}{|c|}{$\Delta\left(t / T_{\mathrm{R}}\right)$} \\
\hline & & 0.9 & 1.5 \\
\hline 2 & 1 & $2.1 \times 10^{-5}$ & $2.6 \times 10^{-5}$ \\
\hline 2 & 10 & $4.4 \times 10^{-6}$ & $1.5 \times 10^{-5}$ \\
\hline 2 & 20 & $3.9 \times 10^{-6}$ & $1.1 \times 10^{-6}$ \\
\hline 2 & 1000 & $2.8 \times 10^{-8}$ & $2.1 \times 10^{-8}$ \\
\hline 10 & 1 & $4.6 \times 10^{-5}$ & $5.9 \times 10^{-5}$ \\
\hline 10 & 10 & $2.2 \times 10^{-4}$ & $3.6 \times 10^{-4}$ \\
\hline 10 & 20 & $1.3 \times 10^{-4}$ & $2.3 \times 10^{-4}$ \\
\hline 10 & 1000 & $1.1 \times 10^{-7}$ & $2.0 \times 10^{-7}$ \\
\hline 50 & 1 & $5.4 \times 10^{-5}$ & $5.2 \times 10^{-5}$ \\
\hline 50 & 10 & $3.6 \times 10^{-4}$ & $5.1 \times 10^{-4}$ \\
\hline 50 & 20 & $5.4 \times 10^{-4}$ & $6.8 \times 10^{-4}$ \\
\hline 50 & 1000 & $3.2 \times 10^{-6}$ & $6.4 \times 10^{-6}$ \\
\hline
\end{tabular}

analysed here decrease with decreasing $N$ (for $N=2$ little change with time range is observed).

\section{APPROXIMATE APPROACHES}

As shown in Section II, it becomes apparent that the sum of two exponentials corresponds to a reasonable representation of the scattering functions for realistic time ranges. Our purpose in this section is to investigate the possibility of obtaining theoretical parameters for this representation from the short-time values of the first three derivatives, avoiding this way the calculation of eigenvalues and eigenvectors. An approximate formula is also derived and investigated.

Actually, the definitions given in eq 22, 24, 31, and 32 , together with eq 39 lead to the equations

$$
\begin{gathered}
P_{1}{ }^{0}+P_{2}{ }^{0}=1 \\
-P_{1}{ }^{0} K_{1}{ }^{0}-P_{2}{ }^{0} K_{2}{ }^{0}=D_{1} \\
P_{1}{ }^{0}\left(K_{1}{ }^{0}\right)^{2}+P_{2}{ }^{0}\left(K_{2}{ }^{0}\right)^{2}=D_{2} \\
-P_{1}{ }^{0}\left(K_{1}{ }^{0}\right)^{3}-P_{2}{ }^{0}\left(K_{2}{ }^{0}\right)^{3}=D_{3}
\end{gathered}
$$

where $P_{1}{ }^{0}, P_{2}{ }^{0}, K_{1}{ }^{0}$, and $K_{2}{ }^{0}$ symbolize the values of the parameters $P_{1}, P_{2}, K_{1}$, and $K_{2}$ obtained from the scattering function in the short-time limit. Therefore, our approach consists in calculating theoretical values of $D_{1}, D_{2}$, and $D_{3}$ from eq 22, 31, and 32 , and also solving the non-linear system
Table V. Values of the parameters $P_{1}{ }^{0}, P_{2}{ }^{0}$,

$K_{1}{ }^{0}$, and $K_{2}{ }^{0}$ obtained from the theoretical first three derivatives of the scattering functions for short times

\begin{tabular}{rrrccr}
\hline$N$ & $x$ & $P_{1}{ }^{0}$ & $P_{2}{ }^{0}$ & $K_{1}{ }^{0}$ & $K_{2}{ }^{0}$ \\
\hline 2 & 1 & 0.980 & 0.020 & 1.028 & 7.406 \\
2 & 10 & 0.633 & 0.367 & 1.394 & 2.498 \\
2 & 20 & 0.618 & 0.382 & 1.554 & 2.290 \\
2 & 1000 & 0.502 & 0.498 & 1.786 & 1.886 \\
& & & & & \\
10 & 1 & 0.998 & 0.002 & 1.096 & 36.802 \\
10 & 10 & 0.908 & 0.092 & 1.685 & 8.018 \\
10 & 20 & 0.769 & 0.231 & 2.048 & 6.076 \\
10 & 1000 & 0.534 & 0.466 & 3.361 & 3.830 \\
& & & & & \\
50 & 1 & 1.000 & - & 1.139 & 234.40 \\
50 & 10 & 0.994 & 0.006 & 2.035 & 40.25 \\
50 & 20 & 0.979 & 0.021 & 2.603 & 26.65 \\
50 & 1000 & 0.429 & 0.571 & 9.035 & 6.742 \\
\hline
\end{tabular}

represented by eq $40-43$ (whose numerical treatment is very simple), and comparing the theoretical parameters so obtained with those calculated by fitting the scattering functions over different time ranges. Table $\mathrm{V}$ summarizes the results for the theoretical parameters.

A comparison between Tables III and V shows that the short-time parameters are dramatically different from those obtained with larger time ranges for high values of $N$. Figures 1 and 2 shows how these differences are propagated to the values of the scattering functions obtained from $P_{1}{ }^{0}, P_{2}{ }^{0}$, $K_{1}{ }^{0}$, and $K_{2}{ }^{0}$ with respect to those calculated directly. It is observed that differences are large in the intermediate region of values for $x$. In fact, the approximate results obtained with the theoretical parameters are comparable in accuracy to those predicted from the initial slope (i.e., just considering the first derivative), for some of the intermediate instances.

We think that the method outlined in this Section is somewhat equivalent to that of Dahler and coworkers ${ }^{13,14}$ who use the technique of calculating memory effects in a projection operator procedure previously proposed by Akcasu et al. ${ }^{23}$ The latter method needs more intricate mathematical treatment, involving complicated expressions. As an advantage Dahler's method is susceptible to general application to detailed polymer structures so that the Rouse-Zimm model corresponds to the simplest 
possible case. In order to analyze the results obtained by both procedures, we calculated some values of our theoretical parameters directly comparable with those plotted in Figures 1 and 2 of ref 14. The comparison, shown in Figures 3 and 4, shows that both sets of results are consistent for small values of the hydrodynamic interaction parameters, though some very remarkable differences are found at higher values of $h^{*}$, except for $K_{2}{ }^{0}$. From the analysis of the results, it may be con-

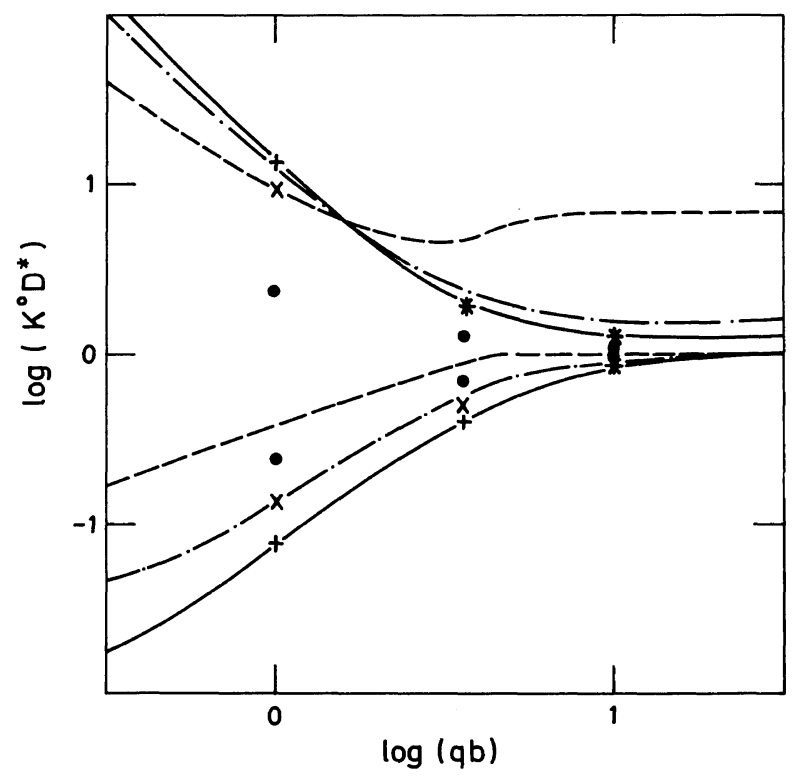

Figure 3. Logarithmic plots of the reduced exponential parameters $K^{0} \equiv K_{1}{ }^{0}$ (below) and $K^{0} \equiv K_{2}{ }^{0}$ (above) vs. qb. The curves represent the results from ref 14 with $h^{*}=0$ (solid curves), $h^{*}=0.1$ (dotted curves), and $h^{*}=0.5$ (broken curves). Our results from the three first derivatives are also represented for $h^{*}=0(+) ; h^{*}=0.1(\times)$ and $h^{*}=0.5(\bullet)$. See text for details.

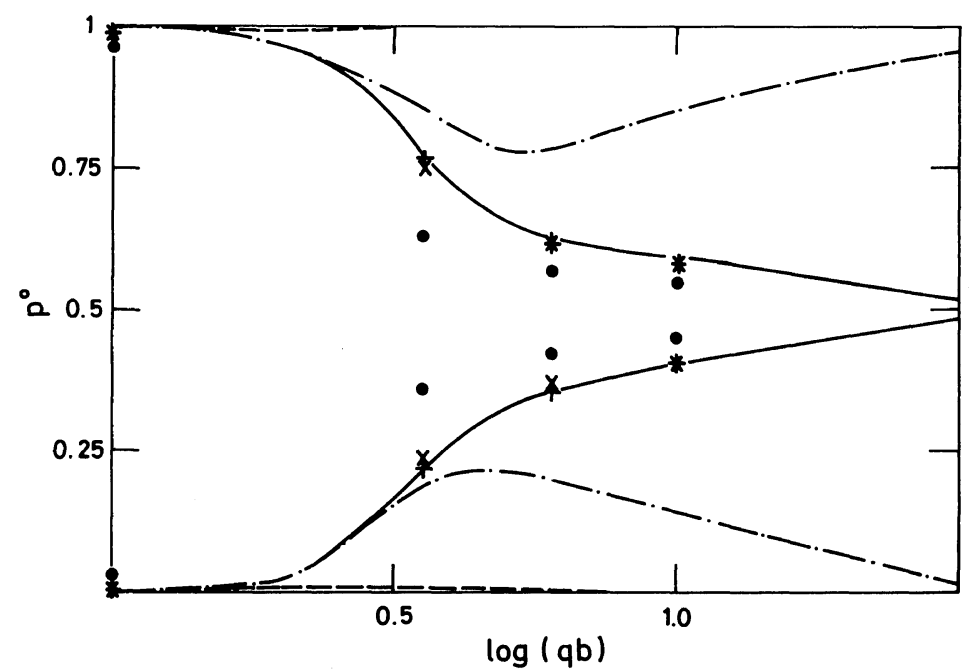

Figure 4. Logarithmic plots of the pre-exponential parameters $P^{0} \equiv P_{1}^{0}$ (above) and $P^{0} \equiv P_{2}^{0}$ (below). See text for details and Figure 3 for other notations. 


\section{J. A. Escudero and J. J. Freire}

cluded that our theoretical parameters are closer to the general trend of the fitted parameters corresponding to the exact scattering functions than those obtained from the projection operator procedure. The most important conclusion is, however, that both methods fail to predict the correct behaviour of the actual functions for realistic time ranges. It seems that both procedures are biased by the short-time approach as is apparent from our numerical calculations reported in Section II, i.e., that the scattering functions exhibit early deviation from the short-time form for most of the intermediate values of $x$.

Very recently, Ou and Dahler, ${ }^{24}$ have proposed a new projection operator procedure based on the use of a set of collective variables which at least in the free draining limit, is able to approximate properly the form of the scattering function.

Their method is promising, since it avoids the triple sum involved in Pecora's expression, eq 5, though it includes the inversion of $(N+1, N+1)$ matrices which, from a computational point of view, is equivalent to calculating the eigenvalues and eigenvectors with eq 6 and hinder the derivation of simpler, analytical formulas. In any case, we feel that more effort should be directed toward establishing a simple theoretical calculation of the scattering function, either from a few theoretical parameters easily computed or deriving a direct double sum expression that can be reduced to an analytical approximate form.

With this goal in mind, we have obtained an approximate theoretical expression for the scattering function. The derivation of this expression starts with eq 18 rearranged in the form

$$
\begin{aligned}
P\left(x, t / T_{\mathrm{R}}\right) & \\
= & (N+1)^{-2} \sum_{i=0}^{N} \sum_{j=0}^{N} \mathrm{e}^{-x|i-j| / N_{\mathrm{G}}} \exp \left\{2\left(x / N_{\mathrm{G}}\right)\right. \\
& \left.\left.\times \sum_{k=0}^{N} \mu_{K}^{-1} Q_{i k} Q_{j k} \exp \left[-\lambda_{k} N_{\mathrm{G}} / 2 x D^{*}\right)\left(t / T_{\mathrm{R}}\right)\right]\right\} .
\end{aligned}
$$

Noticing that

$$
\begin{aligned}
& \left.\sum_{k=0}^{N} \mu_{k}{ }^{-1} Q_{i k} Q_{j k} \exp \left[-\lambda_{k} N_{\mathrm{G}} / 2 x D^{*}\right)\left(t / T_{\mathrm{R}}\right)\right] \\
& \quad=\left\{\boldsymbol{Q} \exp \left[-\left(N_{\mathrm{G}} / 2 x D^{*}\right)\left(t / T_{\mathrm{R}}\right) \boldsymbol{\Lambda}\right] \boldsymbol{M}^{-1} \boldsymbol{Q}^{T}\right\}_{i j}
\end{aligned}
$$

and with the help of eq 7 and 8 , eq 44 can be expressed as

$$
\begin{aligned}
& P\left(x, t / T_{\mathrm{R}}\right)=(N+1)^{-2} \sum_{i=0}^{N} \sum_{j=0}^{N} \mathrm{e}^{-x|i-j| / N_{\mathrm{G}}} \\
& \quad \times \exp \left\{2\left(x / N_{\mathrm{G}}\right)\right\} \\
& \times\left\{\exp \left[-\left(N_{\mathrm{G}} / 2 x D^{*}\right)\left(t / T_{\mathrm{R}}\right) \boldsymbol{H} \boldsymbol{A}\right] \boldsymbol{A}^{-1}-\boldsymbol{A}^{-1}\right\}_{i j} .
\end{aligned}
$$

The series represented by the last exponential on the right side of eq 46 is only convergent for small values of $\left(N_{\mathrm{G}} / x\right)\left(t / T_{\mathrm{R}}\right)$. Thus, the equation can be simplified to,

$P\left(x, t / T_{\mathrm{R}}\right)=(N+1)^{-2} \sum_{i=0}^{N} \sum_{j=0}^{N} \mathrm{e}^{-x|i-j| / N_{\mathrm{G}}} \mathrm{e}^{-\left(H_{i j} / D^{*}\right)\left(t / T_{\mathrm{R}}\right)}$.

The accuracy of this simple formula is satisfactory for most of the realistic range of values of $\left(N_{\mathrm{G}} / x\right)$ $\left(t / T_{\mathrm{R}}\right)$ as can be seen from Figures 1 and 2. In certain cases, employing more terms in the series can be useful. However, these additional terms imply the introduction of positive exponents which in most cases represent a serious disturbance in the convergence, unless a sufficiently high number of terms is considered.

Moreover, it should be remarked that the approximate function would not be adequate in the unrealistic free draining case, for which eq 47 gives a single exponential plus a constant. For the realistic cases studied through this work, the expression is only a poor approximation in the case $x=1$, for the highest values of $\left(t / T_{\mathrm{R}}\right)$. Therefore, we think that eq 47 can be useful in estimating the scattering function for intermediate values of $x / N_{\mathrm{G}}$ which cannot be studied in any other simple way.

\section{REFERENCES}

1. B. J. Berne and R. Pecora, "Dynamic Light Scattering," Wiley, New York, 1971.

2. J. Hendrix, B. Saleh, K. Gnädig, and L. de Maeyer, Polymer, 18, 10 (1977).

3. G. Jones and D. Caroline, Chem. Phys., 37, 187 (1979).

4. I. Nishio and A. Wada, Polym. J., 12, 145 (1980).

5. A. Z. Akcasu, M. Benmouna, and C. C. Han, Polymer, 21, 866 (1980).

6. B. H. Zimm, J. Chem. Phys., 24, 269 (1956).

7. A. Perico, P. Piaggo, and C. Cuniberti, J. Chem. Phys., 62, 2690 (1975).

8. S. C. Lin and J. M. Schurr, Biopolymers, 17, 425 (1978).

9. B. E. A. Saleh and J. Hendrix, Chem. Phys., 12, 25 


\section{Scattering Functions of Polymer Chains}

(1976).

10. J. J. Freire, Polymer, 19, 1441 (1978).

11. J. J. Freire and J. García de la Torre, Chem. Phys., 49, 139 (1980).

12. J. M. Schurr, Chem. Phys., 30, 243 (1978).

13. M. S. Jhon, S. Fesciyan, and J. S. Dahler, J. Polym. Sci., Polym. Phys. Ed., 18, 529 (1980).

14. S. Fesciyan, M. S. Jhon, and J. S. Dahler, J. Polym Sci., Polym. Phys. Ed., 18, 2077 (1980).

15. R. Pecora, J. Chem. Phys., 43, 1562 (1965)

16. H. Yamakawa, "Modern Theory of Polymer Solutions," Harper and Row, New York, 1971.

17. B. H. Zimm, Macromolecules, 13, 592 (1980).

18. A. Perico, P. Piaggo, and C. Cuniberti, J. Chem.
Phys., 62, 4911 (1975).

19. J. T. Fong and A. Peterlin, J. Res. Natl. Bur. Stand. Sect. B, 80, 273 (1976).

20. A. Perico, J. Polym. Sci., Polym. Phys. Ed., 18, 161 (1980).

21. IBM Application Program System/360 (Scientific Subroutine Package), Subroutine NROOT, 1970.

22. J. A. Escudero, Tesis de Licenciatura, Universidad Complutense de Madrid, 1980.

23. A. Z. Akcasu and H. Gurol, J. Polym., Sci. Polym. Phys. Ed., 14, 1 (1976).

24. J. J. Ou, J. J. Dahler, and M. J. Jhon, J. Chem. Phys., 74, 1495 (1981). 